\title{
Evaluación de la calidad del diagnóstico de malaria en la red local de laboratorios y en los laboratorios intermedios en el contexto de la eliminación de la enfermedad en Ecuador
}

\author{
Nohora Marcela Mendoza1, César Eduardo Díaz², Yim Yan Wong³, Adriana Estefanía \\ Echeverría ${ }^{4}$, Doris Grimaneza Guale ${ }^{3}$, Rosa Haydeé Delgado ${ }^{3}$, José Mauricio Muñoz ${ }^{5}$, \\ Luis Fernando Solórzano ${ }^{3}$, Marcelo Eduardo Andrade ${ }^{3}$, Rosa Alba Quintero ${ }^{5}$, Belinda \\ Regina Palacios ${ }^{6}$, Saida Marlene Ortega ${ }^{7}$, Wilson Benigno Cuero ${ }^{5}$, John Richard Castillo ${ }^{5}$ \\ ${ }^{1}$ Corporación para la Investigación y la Innovación, Bogotá, D.C., Colombia \\ ${ }^{2}$ Distrito de Salud 08D01, Coordinación zonal 1, Ministerio de Salud Pública, Esmeraldas, Ecuador \\ ${ }^{3}$ Centro de Referencia Nacional de Parasitología, Instituto Nacional de Investigación en Salud \\ Pública "Leopoldo Izquieta Pérez", Guayaquil, Ecuador \\ ${ }^{4}$ Dirección Nacional de Estrategias de Prevención y Control, Ministerio de Salud Pública, Quito, \\ Ecuador \\ ${ }^{5}$ Coordinación zonal 1, Ministerio de Salud Pública, Esmeralda, Ecuador \\ ${ }^{6}$ Coordinación zonal 8, Ministerio de Salud Pública, Guayaquil, Ecuador \\ ${ }^{7}$ Coordinación zonal 7, Ministerio de Salud Pública, Machala, Ecuador
}

Recibido: $28 / 08 / 18$

Aceptado: $21 / 01 / 19$

Publicado: 30/01/19

\section{Citación:}

Mendoza NM, Díaz CE, Wong YY, Echeverría AE, Guale DG, Delgado RH, et al. Evaluación de la calidad del diagnóstico de malaria en la red local de laboratorios y en los laboratorios intermedios en el contexto de la eliminación de la enfermedad en Ecuador. Biomédica. 2019;39(Supl.2):101-16. https://doi.org/10.7705/biomedica.v39i4.4686

\section{Correspondencia:}

Nohora Marcela Mendoza, Carrera 56 № 167-29, apartamento 204, Bogotá, D.C., Colombia Teléfono: (300) 374 7820; fax: (571) 7596134 marcemendoza07@gmail.com

Contribución de los autores:

Nohora Marcela Mendoza: planeación, elaboración de las bases de datos, análisis de los datos y redacción del manuscrito

Cesar Eduardo Díaz: planeación, análisis de los datos, revisión bibliográfica y redacción de manuscrito

Yim Yam Wong: preparación y aplicación del programa de evaluación externa del desempeño, evaluación de idoneidad, obtención de indicadores y revisión de manuscrito

Adriana Estefanía Echeverría: análisis de los datos, revisión bibliográfica y redacción del manuscrito Doris Grimaneza Guale, Rosa Haydeé Delgado, José Mauricio Muñoz, Marcelo Eduardo Andrade, Rosa Alba Quintero, Belinda Regina Palacios, Saida Marlene Ortega, Wilson Benigno Cuero y John Richard Castillo: evaluación de idoneidad, obtención de indicadores y revisión de manuscrito

Financiación:

Este trabajo fue financiado con recursos de la Organización Panamericana de la Salud y el Ministerio de Salud Pública del Ecuador.

Conflicto de intereses:

Los autores declaran no haber tenido conflictos de intereses de ninguna naturaleza durante las fases de planeación, ejecución, evaluación y análisis, que hubieran podido afectar los resultados de este trabajo.
Introducción. El cumplimiento de la meta de eliminación de la malaria en Ecuador en el 2020 exige contar con la capacidad requerida para el diagnóstico microscópico ajustado a los estándares de calidad de la Organización Mundial de la Salud (OMS) y de la Organización Panamericana de la Salud (OPS) y proveer el tratamiento adecuado a los pacientes.

Objetivo. Conocer la idoneidad o competencia de los microscopistas de la red pública local para el diagnóstico parasitológico de la malaria y el desempeño de los laboratorios intermedios de referencia.

Materiales y métodos. Se hizo un estudio descriptivo de corte transversal a partir de la información obtenida en los talleres de evaluación de idoneidad en el diagnóstico microscópico de la red de laboratorios en las coordinaciones zonales de salud utilizando un panel de láminas para evaluar la concordancia del diagnóstico. Además, se calificó el desempeño de los laboratorios intermedios en el diagnóstico en el marco del programa de evaluación externa del desempeño. Los resultados se compararon con los obtenidos por el laboratorio supranacional de Perú.

Resultados. En los 11 talleres realizados, se evaluó la idoneidad de 191 microscopistas, de los cuales $153(80,1 \%)$ aprobaron las pruebas. Las medianas de los indicadores fueron las siguientes: concordancia entre la detección y el resultado, $100 \%\left(Q_{1}-Q_{3}: 96-100\right)$; concordancia en la especie, $100 \%\left(Q_{1}-Q_{3}: 93-100\right)$; concordancia en el estadio, 93,0\% $\left(Q_{1}-Q_{3}:\right.$ 86-95) y concordancia en el recuento, $77 \%\left(Q_{1}-Q_{3}: 71-82\right)$. En el programa de evaluación externa de desempeño, los tres laboratorios intermedios obtuvieron una concordancia del $100 \%$ en el resultado y una del $96 \%$ en la especie.

Conclusiones. Los indicadores de competencia de la red local y de desempeño de los laboratorios intermedios alcanzaron altos estándares de calidad acordes con el proceso de entrenamiento implementado en el país.

Palabras clave: malaria; control de calidad; diagnóstico; Ecuador; micoscopía; competencias.

Quality evaluation of malaria diagnosis in the local laboratories network and in intermediate laboratories in a setting towards the disease elimination in Ecuador

Introduction: To reach the goal of malaria elimination in Ecuador for the year 2020, it is necessary to have a laboratory network with the capacity to perform microscopic diagnosis according to the WHO/PAHO quality standards and to provide the adequate treatment of cases. Objective: To determine the level of competence for parasitological diagnosis of the microscopists from the local public network and the performance of intermediate reference laboratories.

Materials and methods: We conducted a cross-sectional study based on the information collected in workshops carried out to appraise the competence for microscopic diagnosis of the local laboratory network (zonal health coordinating offices 1 to 8 ) using a slide panel to evaluate diagnosis agreement, as well as the diagnostic performance of the intermediate laboratories using an external quality assessment program. The results were compared against the reference standards of the supranational laboratory in Perú. 
Results: We evaluated the competencies of 191 microscopists in 11 workshops and 153 $(80.1 \%)$ of them were approved. The medians of the indicators were the following: concordance for parasite detection, $100 \%\left(Q_{1}-Q_{3}: 96-100\right)$, concordance for species identification, $100 \%$ $\left(Q_{1}-Q_{3}: 93-100\right)$, and concordances for stage identification, 93.0\% $\left(Q_{1}-Q_{3}: 86-95\right)$ and parasite counting, $77.0 \%\left(Q_{1}-Q_{3}: 71-82\right)$. In the external quality assessment, the three intermediate laboratories obtained $100 \%$ in parasite detection concordance and $96 \%$ for species detection concordance.

Conclusions: The results for the primary network and the performance indicators for the intermediate laboratories showed the high-quality standards of the training program implemented in the country.

Keywords. Malaria; quality control; diagnosis; Ecuador; microscopy; competency.

En Ecuador se han hecho importantes esfuerzos para el control de la malaria, o paludismo, los cuales se han visto reflejados en el descenso del número de casos desde el 2002. Sin embargo, según el informe de casos reportados por provincia entre el 2014 y el 2017, recolectado en el Sistema de Vigilancia Epidemiológica de Malaria del Ecuador (Sivemae), ha habido un incremento de casos ocasionados por las dos especies circulantes, Plasmodium vivax y $P$. falciparum. De todas maneras, el país ha logrado convertirse en uno de los 21 países que ya marchan hacia la eliminación de la malaria (1).

Simultáneamente con el cierre del antiguo Servicio Nacional de Control de Enfermedades Transmitidas por Vectores Artrópodos (2), el Ministerio de Salud Pública de Ecuador, en coordinación con la Organización Panamericana de la Salud (OPS), inició la planeación estratégica, la estandarización, el fortalecimiento técnico y el posicionamiento del Instituto Nacional de Investigación en Salud Pública y de los laboratorios intermedios, con el fin de dotarlos de los conocimientos necesarios para liderar la red de diagnóstico de malaria.

La estructura de la red incluye el nivel nacional, con el Laboratorio de Referencia Nacional que hace parte del Instituto Nacional de Investigación en Salud Pública (3), el nivel intermedio, conformado por tres laboratorios ubicados en El Oro, Esmeraldas y Guayas, y el nivel local, representado por los laboratorios de análisis clínico del sector público.

En términos epidemiológicos, la falta de un diagnóstico efectivo se traduce en el mantenimiento de la transmisión de la enfermedad en lugares receptivos y, en términos de la atención al paciente, significa un aumento de la probabilidad de complicaciones e, incluso, de la muerte por paludismo (4).

Según los lineamientos internacionales, los casos de malaria deben ser confirmados por pruebas de diagnóstico para iniciar un esquema de tratamiento radical con antimaláricos efectivos (5). En consecuencia, es necesario garantizar la eficacia del diagnóstico, aunque para ello haya que enfrentar múltiples factores: presencia de diferentes especies y estadios parasitarios, endemia de la enfermedad, familiaridad del personal de laboratorio con la morfología del parásito o con los procedimientos de diagnóstico y su aplicación rigurosa, disponibilidad de los equipos, insumos y reactivos básicos en la red de laboratorios para garantizar exámenes de calidad, así como los aspectos relacionados con la carga laboral y la ausencia de sospecha frente a casos probables por el nexo clínico y epidemiológico, entre otros (4-6).

Los países que emprenden el proceso de eliminación de la malaria deben contar con un sistema de vigilancia y respuesta de buena calidad que permita sospechar prontamente, detectar oportunamente, diagnosticar con 
calidad, brindar tratamiento rápido y efectivo, hacer el seguimiento de los casos positivos e iniciar la investigación en busca de más casos. Además del diagnóstico de calidad, los países requieren el desarrollo y fortalecimiento de una red de laboratorios locales y de referencia con capacidad para la verificación parasitológica (7).

El método de diagnóstico parasitológico de rutina y de referencia de la malaria continúa siendo la microscopía de la gota gruesa y del extendido $(6,8)$. Como complemento, es posible utilizar las pruebas de diagnóstico rápido en aquellos sitios de difícil acceso y en los establecimientos de salud que tengan horarios restringidos, o donde no sea factible la implementación de la microscopía para mejorar la oportunidad del diagnóstico de los pacientes con malaria $(9,10)$. Sin embargo, cuando ya se avizora la eliminación, la detección de las parasitemias muy bajas y submicroscópicas representa un reto en el que el diagnóstico mediante las técnicas de biología molecular tiene un papel relevante $(11,12)$.

En los laboratorios que ofrecen el servicio de diagnóstico de malaria debe haber personal capacitado en todos los procedimientos relacionados con el diagnóstico y el control de calidad $(6,13)$. En ese sentido, el Ministerio de Salud Pública de Ecuador, el Instituto Nacional de Investigación en Salud Pública y la OPS trabajaron de forma coordinada para actualizar los procesos de control de calidad del diagnóstico parasitológico, de manera que el país contara con lineamientos ajustados a los estándares internacionales (6).

Con el apoyo de la OPS, el Instituto Nacional de Investigación en Salud Pública capacitó al personal de los laboratorios intermedios y locales, en estos últimos con una periodicidad anual durante tres años consecutivos, en los lineamientos técnicos, familiarizando a los microscopistas con el diagnóstico parasitológico en un contexto de baja transmisión de malaria.

Como parte del apoyo brindado por la OPS a la red nacional de laboratorios para fortalecer el programa nacional de malaria, entre julio y agosto del 2017 se organizaron 11 talleres de reentrenamiento en ocho de las nueve zonas establecidas por el Ministerio de Salud Pública dirigidos a los laboratorios de análisis clínico del sector público encargados del diagnóstico de malaria, con el fin de evaluar la idoneidad del diagnóstico microscópico. Asimismo, en febrero de 2018 el Instituto Nacional de Investigación en Salud Pública, con apoyo de la OPS, organizó la capacitación en el programa de evaluación externa del desempeño para los referentes de los laboratorios intermedios, con el fin de conocer su desempeño en el diagnóstico de malaria.

\section{Materiales y métodos}

\section{Tipo de estudio}

Se hizo un estudio descriptivo de corte transversal para conocer la idoneidad de los microscopistas del nivel local en el diagnóstico de malaria y el desempeño de los referentes del nivel intermedio.

\section{Caracterización sociodemográfica de los microscopistas}

A los microscopistas del sector público en el nivel local de las zonas 1 a 8 se les solicitaron los datos referentes a la edad, el nivel educativo, el sitio de trabajo y la experiencia, para contar con una base de datos más completa de los integrantes de la red, útil para su análisis y como insumo para el nivel intermedio y el nacional. 


\section{Evaluación de idoneidad}

En los talleres de actualización de conocimientos y evaluación de la idoneidad para el fortalecimiento del diagnóstico microscópico participaron los responsables de esta actividad en el nivel local.

Los talleres se desarrollaron durante cinco días, tres días para la actualización y dos para la evaluación de la competencia. Se evaluó el componente teórico mediante pruebas antes y después de la capacitación, las cuales contenían 25 preguntas sobre el vector de la enfermedad, el diagnóstico parasitológico, las actividades de control de calidad, y los aspectos clínicos generales y del tratamiento de la enfermedad; este componente no afectó la evaluación de la idoneidad. Se hizo una prueba práctica inicial para contar con un punto de partida que sirviera de guía para la actualización.

La evaluación de la idoneidad se hizo utilizando paneles de 24 láminas (muestras positivas y negativas para malaria) donadas por el laboratorio supranacional que opera desde el Instituto Nacional de Salud de Perú (Programa de Evaluación Externa del Desempeño Regional de OPS) y preparados por el Instituto Nacional de Investigación en Salud Pública. El tiempo de lectura por lámina fue de 10 minutos.

\section{Evaluación del desempeño}

Se evaluó el desempeño de los referentes de los laboratorios intermedios con un panel de 20 láminas (muestras positivas y negativas), uno para cada uno, los cuales fueron donados por el laboratorio supranacional en Perú y preparados por el Instituto Nacional de Investigación en Salud Pública. Se distribuyeron paneles recodificados a los participantes para que fueran leídos en sus lugares de trabajo.

Criterios de lectura para la gota gruesa

- Muestra negativa: no se observan formas parasitarias de Plasmodium spp., en 500 campos observados con un objetivo de 100X.

- Muestra positiva: se observan formas parasitarias de Plasmodium spp. En este caso, era necesario informar sobre la especie o especies presentes y reportar la densidad parasitaria en parásitos $/ \mu$, teniendo en cuenta los siguientes criterios:

$$
\begin{aligned}
& \text { Densidad parasitaria }=\frac{\leq 9 \text { parásitos } \times 6.000 \text { leucocitos } / \mu \mathrm{l} \text { de sangre }}{500 \text { leucocitos contados }} \\
& \text { Densidad parasitaria }=\frac{\geq 10 \text { parásitos } \times 6.000 \text { leucocitos } / \mu \text { de sangre }}{200 \text { leucocitos contados }} \\
& \text { Densidad parasitaria }=\frac{\geq 500 \text { parásitos } \times 6.000 \text { leucocitos } / \mu \mathrm{l} \text { de sangre }}{\text { número de leucocitos contados }}
\end{aligned}
$$

\section{Indicadores de las evaluaciones}

Los indicadores se determinaron con referencia a los resultados del laboratorio supranacional en cuanto a la detección del parásito, la especie y la densidad parasitaria por estadio.

Se determinaron los siguientes indicadores para cada participante, tanto en la evaluación de la idoneidad del nivel local como en la evaluación del desempeño del nivel intermedio.

Concordancia de resultado (detección del parásito): se evaluó la capacidad de discriminar la presencia o ausencia de formas parasitarias 
de Plasmodium spp. y, para el cálculo, se compararon los resultados del personal capacitado con los del laboratorio de referencia.

Concordancia de especie (diferenciación de especie): se evaluó el reconocimiento de cada especie parasitaria en las láminas positivas y, para el cálculo, se compararon los resultados del personal capacitado y los del referente.

Concordancia de estadio: se determinó el reconocimiento de estadios sexuados y asexuados de Plasmodium spp. en las láminas positivas. El cálculo se obtuvo comparando los resultados del personal capacitado con los del laboratorio referente.

Concordancia de recuento (densidad parasitaria): se evaluó la capacidad para contar la cantidad de parásitos por microlitro $(\mu l)$ de sangre y la concordancia con la densidad parasitaria del laboratorio referente. Se consideró como concordante un recuento que no tuviera una diferencia mayor de $\pm 50 \%$ con respecto al del laboratorio referente.

Posteriormente, se obtuvo el puntaje de concordancia de cada muestra, para luego aplicar la fórmula general de concordancia. Se estimó como puntaje satisfactorio uno de $80 \%$ o más muestras concordantes con las del evaluador.

Los indicadores se calcularon aplicando la siguiente fórmula (14-16):

$$
\text { Concordancia }=\frac{\text { Puntaje obtenido }}{\text { Puntaje ideal }} \times 100
$$

Además, para la evaluación de la idoneidad se determinaron la sensibilidad, la especificidad y el índice kappa, en tanto que para la evaluación del desempeño, se estimaron el índice kappa y los valores predictivos $(6,14)$. Estos indicadores se calcularon con base en los resultados de los microscopistas comparados con los del laboratorio supranacional.

\section{Paneles de láminas}

Se utilizaron dos páneles.

Panel de evaluación del desempeño del nivel local o red primaria. El panel estaba compuesto por 24 láminas (10 negativas y 14 positivas); las muestras positivas incluían 10 láminas positivas para $P$. falciparum, 3 de ellas con parasitemia entre 100 y 300 parásitos/ $\mu l ; 4$ láminas con parasitemias entre

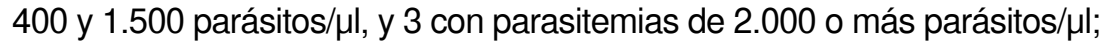
por otra parte, 3 láminas eran positivas para $P$. vivax, una con densidad parasitaria entre 100 y 200 parásitos/ $\mu$ l, otra con densidad parasitaria entre 500 y 600 parásitos $/ \mu \mathrm{l}$, y una última con densidad parasitaria entre 8.000 y 12.000 parásitos/ $/$ l. Asímismo, se incluyó una lámina con muestra mixta $(P$. falciparum y $P$. vivax) (6).

Panel para la evaluación del desempeño del nivel intermedio. Este incluyó 20 láminas. La composición del panel fue la siguiente: 6 muestras negativas; 6 muestras positivas para $P$. falciparum: 4 con una parasitemia entre 70 y 200 parásitos/ $\mu$ l, y 2 con parasitemia entre 300 y 500 parásitos $/ \mu$; por otro parte, se incluyeron 4 muestras positivas para $P$. vivax: 2 con parasitemia entre 70 y 200 parásitos/ $\mu \mathrm{l}$ y 2 con parasitemia entre 400 y 700 parásitos $/ \mu \mathrm{l}$; por último, 4 muestras de infección mixta $(P$. falciparum $+P$. vivax).

Los paneles utilizados siguieron los lineamientos de la Organización Mundial de la Salud (OMS) (6). 


\section{Lugar y periodo de estudio}

Los 11 talleres contaron con participantes de las zonas de salud 1 a 8 establecidas por el Ministerio de Salud Pública y se realizaron del 17 de julio al 11 de agosto del 2017 en las ciudades de Macas, Machala, Esmeraldas, Portoviejo y Guayaquil. El panel de láminas para la evaluación de la idoneidad de los laboratorios intermedios se envió a cada laboratorio en las ciudades de Machala, Guayaquil y Esmeraldas en marzo del 2018.

\section{Procedimiento}

Evaluación de la idoneidad del nivel local. El Ministerio de Salud Pública convocó a 208 microscopistas con experiencia en el diagnóstico de malaria, responsables de esta labor en el nivel local. Se hicieron 11 talleres teóricoprácticos de actualización del conocimiento dirigidos por tres o cuatro facilitadores del nivel nacional, zonal, local y un experto internacional. Los resultados de las evaluaciones se ingresaron a la base de datos que contenía la información de los participantes.

La evaluación teórica se consideró aprobada con 80 \% o más respuestas correctas. Por otra parte, las concordancias de resultado y especie se consideraron satisfactorias al obtener $90 \%$ o más aciertos; estos porcentajes fueron los puntajes mínimos de aprobación de las pruebas. Las concordancias de estadio y recuento se consideraron satisfactorias al obtener $80 \%$ o más aciertos. A cada participante se le entregó un certificado de aprobación del taller o, en su defecto, un certificado de participación.

Programa de evaluación externa del desempeño. Esta actividad se hizo simultáneamente en los tres laboratorios intermedios, los cuales son responsables de hacer el control de calidad de los laboratorios del nivel local. El Instituto Nacional de Investigación en Salud Pública envió los paneles acompañados de instrucciones y registros de las respuestas. Se dieron 10 días para reportar los resultados. Los valores de los indicadores de esta actividad y las acciones propuestas en cada caso se presentan en el cuadro 1 (14).

Cuadro 1. Interpretación y acciones requeridas con base en los indicadores de concordancia del programa de evaluación externa del desempeño

\begin{tabular}{lcll}
\hline Indicador & $(\%)$ & Interpretación & Acción requerida \\
\hline Concordancia de resultado & $95-100$ & Satisfactorio & Continuar con el PEED \\
& $85-<95$ & Cuestionable & Supervisión \\
& $<85$ & Insatisfactorio & Actualización del entrenamiento \\
Concordancia de especie & $95-100$ & Satisfactorio & Continuar con el PEED \\
& $85-<95$ & Cuestionable & Supervisión \\
Concordancia de estadio & $<85$ & Insatisfactorio & Actualización del entrenamiento \\
& $80-100$ & Satisfactorio & Continuar con el PEED \\
& $70-<80$ & Cuestionable & Supervisión \\
Concordancia de parasitemia* & $80-100$ & Satisfactorio & Continuar con el PEED \\
& $60-<80$ & Cuestionable & Supervisión \\
& $<60$ & Insatisfactorio & Actualización del entrenamiento \\
\hline
\end{tabular}

PEED: programa de evaluación externa del desempeño

*Se considera concordante una muestra sin diferencia de más del $50 \%$ de la parasitemia con respecto al laboratorio de referencia, es decir, se acepta un margen de error de $\pm 50 \%$ en la parasitemia de la muestra, para luego aplicar la fórmula general de concordancia aceptando como satisfactorio un puntaje $\geq 80 \%$ con respecto al puntaje ideal del evaluador. 


\section{Análisis estadístico}

Los datos obtenidos fueron tabulados y analizados con Excel 16.0 ${ }^{\mathrm{TM}}$ y, además, la información fue exportada y analizada con los programas IBM SPSS Statistics ${ }^{\mathrm{TM}}$, versión 24 para Windows y Stata $11.1^{\mathrm{TM}}$.

Se determinaron frecuencias absolutas, proporciones, promedios, medianas, intervalos de confianza (IC) del $95 \%$ y rangos intercuartílicos (RIC: primer y tercer cuartiles). Se calcularon las significaciones estadísticas de las evaluaciones en que se hicieron las pruebas antes y después de la capacitación: componentes teórico y práctico para las variables de concordancia de resultado y de especie utilizando la prueba de ji al cuadrado con un valor de $p<0,05$ como significativo.

Se estimaron las concordancias de resultado, especie, estadio y parasitemia, así como la sensibilidad y la especificidad con sus intervalos de confianza del $95 \%$. Los resultados de los indicadores de la evaluación de los participantes en el estudio se compararon con los resultados de las lecturas del laboratorio supranacional de referencia.

\section{Aspectos éticos}

Las láminas fueron obtenidas de pacientes con malaria y sanos, quienes previamente firmaron un consentimiento informado para el uso de las muestras. El Instituto Nacional de Salud de Perú cumplió con los protocolos internacionales para la elaboración del banco de láminas (6) y mantuvo la confidencialidad de los pacientes con malaria asignando un código a las láminas enviadas. Asimismo, el Instituto Nacional de Investigación en Salud Pública mantuvo la confidencialidad de los datos de los pacientes y de los resultados de los participantes utilizando láminas codificadas, en cumplimiento del Artículo 7 del acuerdo ministerial № 5216 del 2014.

\section{Resultados}

\section{Red de microscopía local}

Asistieron 191 participantes, es decir, el $92 \%$ de los microscopistas convocados. De estos, 50 (26\%) provenían de la zona 1, 41 (22\%) de la zona 4, 29 (15\%) de la zona 5, $23(12 \%)$ de la zona 8, 17 (9\%) de la zona 7, $12(6 \%)$ de la zona 6, $12(6 \%)$ de la zona 2 y 7 (4\%) de la zona 3.

El grupo de microscopistas capacitados del nivel local estaba conformado por 104 mujeres y 87 hombres, con un tiempo promedio de 14 años de experiencia en el diagnóstico de malaria; 185 (96,8\%) participantes informaron tener dos 0 más años de servicio. El tiempo promedio desde la última capacitación fue de 1,9 años; 107 (56\%) participantes indicaron haber sido entrenados en el último año, 50 (26\%) más de un año atrás, 33 (17\%) no dieron esta información y uno (1\%) refirió no haber sido entrenado. El nivel educativo se discriminó así: educación básica, 8 participantes (4\%), secundaria, 117 (61\%), tecnológica, 2 ( $1 \%)$, y superior, 64 (34\%); sin embargo, solo $9(14 \%)$ de los participantes con educación superior tenían grado profesional relacionado con el área de la salud. La información sobre las características sociodemográficas de los microscopistas según la zona se presenta en el cuadro 2.

El porcentaje de aciertos en la prueba teórica previa tuvo una mediana de $56 \%\left(Q_{1}-Q_{3}: 44-68 \% ; R I C=24\right)$, en tanto que en la prueba posterior se obtuvo una mediana de $96,0 \%\left(Q_{1}-Q_{3}: 88-100 \%\right.$; $\left.R I C=12\right)$. 
Cuadro 2. Características sociodemográficas de los microscopistas del nivel local

\begin{tabular}{|c|c|c|c|c|c|c|c|c|c|c|c|c|}
\hline \multirow[b]{2}{*}{ Zona } & \multicolumn{2}{|c|}{ Nivel de atención } & \multirow{2}{*}{$\begin{array}{l}\text { Laboratorio } \\
\text { intermedio }\end{array}$} & \multirow{2}{*}{$\begin{array}{l}\text { Edad } \\
\text { promedio } \\
\text { (años) }\end{array}$} & \multicolumn{2}{|c|}{ Sexo } & \multirow{2}{*}{$\begin{array}{l}\text { Experiencia } \\
\text { promedio } \\
\text { (años) }\end{array}$} & \multirow{2}{*}{$\begin{array}{l}\text { Tiempo promedio } \\
\text { desde la última } \\
\text { capacitación (años) }\end{array}$} & \multicolumn{4}{|c|}{ Nivel de educación } \\
\hline & Primero & Segundo & & & $\mathbf{F}$ & $\mathbf{M}$ & & & EB & $\mathrm{Sec}$ & Tec & Sup \\
\hline Zona 1 & 44 & 6 & 0 & 44 & 22 & 28 & 15 & 2 & 1 & 44 & 1 & 4 \\
\hline Zona 2 & 11 & 1 & 0 & 44 & 6 & 6 & 13 & 3 & 0 & 10 & 0 & 2 \\
\hline Zona 3 & 6 & 1 & 0 & 42 & 1 & 6 & 12 & 2,5 & 2 & 4 & 0 & 1 \\
\hline Zona 4 & 36 & 5 & 0 & 41 & 24 & 17 & 15 & 1 & 0 & 23 & 0 & 18 \\
\hline Zona 5 & 16 & 13 & 0 & 44 & 25 & 4 & 14 & 2 & 0 & 9 & 1 & 19 \\
\hline Zona 6 & 9 & 3 & 0 & 41 & 1 & 11 & 9 & 2 & 5 & 6 & 0 & 1 \\
\hline Zona 7 & 10 & 7 & 0 & 45 & 6 & 11 & 15 & 1,5 & 0 & 7 & 0 & 10 \\
\hline Zona 8 & 14 & 7 & 2 & 44 & 19 & 4 & 15 & 1 & 0 & 14 & 0 & 9 \\
\hline
\end{tabular}

F: femenino; M: masculino; EB: educación básica; Sec: secundaria; Tec: tecnología; Sup: superior

En la evaluación práctica, para la concordancia de resultado se obtuvo una mediana de $90 \%\left(Q_{1}-Q_{3}: 90-100 \%\right)$ en la prueba previa, en tanto que en la prueba posterior (evaluación de idoneidad) la concordancia de resultado tuvo una mediana de $100 \%\left(Q_{1}-Q_{3}: 96-100 \%\right)$.

En cuanto a la concordancia de especie, en la prueba previa la mediana fue de $86,1 \%\left(Q_{1}-Q_{3}: 71-93 \%\right)$ y, en la posterior (evaluación de idoneidad), de $100 \%\left(Q_{1}-Q_{3}: 93-100 \%\right)$.

Las concordancias de estadio y recuento solamente fueron consideradas para la evaluación de idoneidad; para la concordancia de estadio la mediana fue de $93 \%$ $\left(Q_{1}-Q_{3}: 86-95 \%\right)$ y, para la de recuento, esta fue de $77 \%\left(Q_{1}-Q_{3}: 71-82 \%\right)$.

En el cuadro 3 se presenta el número de participantes con evaluaciones aprobadas y desaprobados en ambas pruebas, así como las variables de resultado y especie antes y después de la capacitación con su significación estadística (ji al cuadrado).

Cuadro 3. Número de participantes aprobados y no aprobados en las pruebas anteriores y posteriores al entrenamiento de los microscopistas del nivel local por zona, 2017

\begin{tabular}{|c|c|c|c|c|c|c|c|c|c|c|c|}
\hline \multirow{3}{*}{ Zonas } & \multirow{3}{*}{$\mathbf{n}$} & \multirow{3}{*}{$\begin{array}{l}\text { Momento } \\
\text { de la } \\
\text { prueba }\end{array}$} & \multirow{2}{*}{\multicolumn{2}{|c|}{ Prueba teórica }} & \multirow{3}{*}{$\mathbf{p}$} & \multicolumn{6}{|c|}{ Prueba práctica } \\
\hline & & & & & & \multicolumn{3}{|c|}{ Resultado } & \multicolumn{3}{|c|}{ Especie } \\
\hline & & & $\begin{array}{l}\text { Número } \\
\text { aprobado }\end{array}$ & $\begin{array}{c}\text { Número no } \\
\text { aprobado }\end{array}$ & & $\begin{array}{l}\text { Número } \\
\text { aprobado }\end{array}$ & $\begin{array}{l}\text { Número no } \\
\text { aprobado }\end{array}$ & p & $\begin{array}{l}\text { Número } \\
\text { aprobado }\end{array}$ & $\begin{array}{l}\text { Número no } \\
\text { aprobado }\end{array}$ & p \\
\hline Zona 1 & 50 & $\begin{array}{l}\text { Previa } \\
\text { Posterior }\end{array}$ & $\begin{array}{r}6 \\
50\end{array}$ & $\begin{array}{r}44 \\
0\end{array}$ & $<0,05$ & $\begin{array}{l}44 \\
50\end{array}$ & $\begin{array}{l}6 \\
0\end{array}$ & $<0,05$ & $\begin{array}{l}28 \\
49\end{array}$ & $\begin{array}{r}22 \\
1\end{array}$ & $<0,05$ \\
\hline Zona 2 & 12 & $\begin{array}{l}\text { Previa } \\
\text { Posterior }\end{array}$ & $\begin{array}{r}0 \\
11\end{array}$ & $\begin{array}{r}12 \\
1\end{array}$ & $<0,05$ & $\begin{array}{l}12 \\
12\end{array}$ & $\begin{array}{l}0 \\
0\end{array}$ & $>0,05$ & $\begin{array}{r}8 \\
12\end{array}$ & $\begin{array}{l}4 \\
0\end{array}$ & $<0,05$ \\
\hline Zona 4 & 41 & $\begin{array}{l}\text { Previa } \\
\text { Posterior }\end{array}$ & $\begin{array}{l}13 \\
40\end{array}$ & $\begin{array}{r}28 \\
1\end{array}$ & $<0,05$ & $\begin{array}{l}35 \\
39\end{array}$ & $\begin{array}{l}6 \\
2\end{array}$ & $>0,05$ & $\begin{array}{l}13 \\
28\end{array}$ & $\begin{array}{l}28 \\
13\end{array}$ & $<0,05$ \\
\hline Zona 5 & 29 & $\begin{array}{l}\text { Previa } \\
\text { Posterior }\end{array}$ & $\begin{array}{r}1 \\
19\end{array}$ & $\begin{array}{l}28 \\
10\end{array}$ & $<0,05$ & $\begin{array}{r}9 \\
29\end{array}$ & $\begin{array}{r}20 \\
0\end{array}$ & $<0,05$ & $\begin{array}{r}4 \\
18\end{array}$ & $\begin{array}{l}25 \\
11\end{array}$ & $<0,05$ \\
\hline Zona 6 & 12 & $\begin{array}{l}\text { Previa } \\
\text { Posterior }\end{array}$ & $\begin{array}{l}0 \\
4\end{array}$ & $\begin{array}{r}12 \\
8\end{array}$ & $<0,05$ & $\begin{array}{l}10 \\
11\end{array}$ & $\begin{array}{l}2 \\
1\end{array}$ & $>0,05$ & $\begin{array}{r}4 \\
11\end{array}$ & $\begin{array}{l}8 \\
1\end{array}$ & $<0,05$ \\
\hline Zona 8 & 23 & $\begin{array}{l}\text { Previa } \\
\text { Posterior }\end{array}$ & $\begin{array}{r}2 \\
21\end{array}$ & $\begin{array}{r}21 \\
2\end{array}$ & $<0,05$ & $\begin{array}{l}16 \\
23\end{array}$ & $\begin{array}{l}7 \\
0\end{array}$ & $<0,05$ & $\begin{array}{r}8 \\
18\end{array}$ & $\begin{array}{r}15 \\
5\end{array}$ & $<0,05$ \\
\hline
\end{tabular}

$n$ : número de participantes; $p \leq 0,05$ : diferencia estadísticamente significativa 
Por otra parte, al comparar las lecturas de los participantes con las del laboratorio supranacional de referencia en la evaluación de la idoneidad, para la sensibilidad se obtuvo una mediana de $100 \%\left(Q_{1}-Q_{3}: 100-100 \%\right)$ y, para la especificidad, una de $100 \%\left(Q_{1}-Q_{3}: 100-100 \%\right)$. El índice kappa general tuvo un valor mínimo de 0,14 y un máximo de 1,0, en tanto que el índice kappa de especie arrojó un valor mínimo de 0,10 y un máximo de 1,0. El consolidado nacional de los indicadores de la evaluación de competencias se presenta en el cuadro 4.

En la evaluación de idoneidad, $153(80,1 \%)$ participantes aprobaron las pruebas y 38 (19,9\%) no lo hicieron (figura 1). El total de errores en $77(40 \%)$ de los participantes fue de 192 en las concordancias de resultado y de especie, de 81 (42\%) en la diferenciación entre láminas positivas y negativas y de 111 (58\%) en la diferenciación de especies; en este último aspecto, 74 (67\%) correspondieron a la identificación errada de $P$. falciparum como $P$. vivax, 19 (17\%) a identificaciones de $P$. vivax como $P$. falciparum, $8(7 \%)$ a diagnósticos errados de infección mixta como $P$. falciparum, $6(5 \%)$ a confusión de $P$. vivax con infección mixta y $4(4 \%)$ a fallas al identificar infección mixta como de $P$. vivax.

\section{Programa de evaluación externa del desempeño del nivel intermedio}

Los resultados de la evaluación del desempeño de los tres laboratorios intermedios de referencia se observan en el cuadro 5. La deficiencia en el diagnóstico en estos tres laboratorios consistió en clasificar una infección mixta ( $P$. falciparum y $P$. vivax) como infección por $P$. falciparum.

\section{Discusión}

Entre las actividades para garantizar la calidad del diagnóstico de malaria, se encuentra el entrenamiento en el diagnóstico microscópico con evaluación de la idoneidad, mecanismo por el cual los microscopistas nuevos reciben lineamientos del programa y adquieren habilidades y conocimientos

Cuadro 4. Consolidado nacional de los indicadores de la evaluación de idoneidad de los microscopistas

\begin{tabular}{|c|c|c|c|c|c|c|c|c|}
\hline \multirow[b]{2}{*}{ Zona/país } & \multicolumn{4}{|c|}{ Mediana de concordancias (\%) } & \multirow[b]{2}{*}{ Sensibilidad (\%) } & \multirow[b]{2}{*}{ Especificidad (\%) } & \multicolumn{2}{|c|}{ Intervalos } \\
\hline & $\begin{array}{c}\text { Resultado } \\
\left(Q_{1}-Q_{3}\right)\end{array}$ & $\begin{array}{c}\text { Especie } \\
\left(Q_{1}-Q_{3}\right)\end{array}$ & $\begin{array}{l}\text { Estadio } \\
\left(Q_{1}-Q_{3}\right)\end{array}$ & $\begin{array}{c}\text { Recuento } \\
\left(Q_{1}-Q_{3}\right)\end{array}$ & & & IKG & IKE \\
\hline Zona 1 & $\begin{array}{c}100 \\
(100-100)\end{array}$ & $\begin{array}{c}100 \\
(100-100)\end{array}$ & $\begin{array}{c}93 \\
(93-95)\end{array}$ & $\begin{array}{c}79 \\
(75-82)\end{array}$ & $\begin{array}{c}100 \\
(100-100)\end{array}$ & $\begin{array}{c}100 \\
(100-100)\end{array}$ & $0,93-1,0$ & $0,69-1,0$ \\
\hline Zona 2 & $\begin{array}{c}100 \\
(100-100)\end{array}$ & $\begin{array}{c}100 \\
(95-100)\end{array}$ & $\begin{array}{c}93 \\
(87-95)\end{array}$ & $\begin{array}{c}81 \\
(76-84)\end{array}$ & $\begin{array}{c}100 \\
(100-100)\end{array}$ & $\begin{array}{c}100 \\
(100-100)\end{array}$ & 1,0 & $0,85-1,0$ \\
\hline Zona 3 & $\begin{array}{c}100 \\
(96-100)\end{array}$ & $\begin{array}{c}100 \\
(82-100)\end{array}$ & $\begin{array}{c}91 \\
(79-100)\end{array}$ & $\begin{array}{c}77 \\
(66-80)\end{array}$ & $\begin{array}{c}100 \\
(100-100)\end{array}$ & $\begin{array}{c}100 \\
(90-100)\end{array}$ & $0,83-1,0$ & $0,55-1,0$ \\
\hline Zona 4 & $\begin{array}{c}100 \\
(100-100)\end{array}$ & $\begin{array}{c}100 \\
(86-100)\end{array}$ & $\begin{array}{c}93 \\
(82-96)\end{array}$ & $\begin{array}{c}75 \\
(69-82)\end{array}$ & $\begin{array}{c}100 \\
(100-100)\end{array}$ & $\begin{array}{c}100 \\
(100-100)\end{array}$ & $0,14-1,0$ & $0,10-1,0$ \\
\hline Zona 5 & $\begin{array}{c}100 \\
(96-100)\end{array}$ & $\begin{array}{c}93 \\
(86-100)\end{array}$ & $\begin{array}{c}86 \\
(81-94)\end{array}$ & $\begin{array}{c}75 \\
(67-79)\end{array}$ & $\begin{array}{c}100 \\
(97-100)\end{array}$ & $\begin{array}{c}100 \\
(90-100)\end{array}$ & $0,82-1,0$ & $0,22-1,0$ \\
\hline Zona 6 & $\begin{array}{c}100 \\
(94-100)\end{array}$ & $\begin{array}{c}100 \\
(93-100)\end{array}$ & $\begin{array}{c}91 \\
(87-95)\end{array}$ & $\begin{array}{c}79 \\
(71-84)\end{array}$ & $\begin{array}{c}100 \\
(100-100)\end{array}$ & $\begin{array}{c}100 \\
(93-100)\end{array}$ & $0,83-1,0$ & $0,75-1,0$ \\
\hline Zona 7 & $\begin{array}{c}96 \\
(92-98)\end{array}$ & $\begin{array}{c}100 \\
(90-100)\end{array}$ & $\begin{array}{c}91 \\
(84-93)\end{array}$ & $\begin{array}{c}75 \\
(70-79)\end{array}$ & $\begin{array}{c}100 \\
(100-100)\end{array}$ & $\begin{array}{c}90 \\
(80-95)\end{array}$ & $0,64-1,0$ & $0,71-1,0$ \\
\hline Zona 8 & $\begin{array}{c}100 \\
(96-100)\end{array}$ & $\begin{array}{c}100 \\
(93-100)\end{array}$ & $\begin{array}{c}91 \\
(84-93)\end{array}$ & $\begin{array}{c}77 \\
(71-82)\end{array}$ & $\begin{array}{c}100 \\
(100-100)\end{array}$ & $\begin{array}{c}100 \\
(100-100)\end{array}$ & $0,82-1,0$ & $0,52-1,0$ \\
\hline $\begin{array}{l}\text { País } \\
\text { (consolidado) }\end{array}$ & $\begin{array}{c}100 \\
(96-100)\end{array}$ & $\begin{array}{c}100 \\
(93-100)\end{array}$ & $\begin{array}{c}93 \\
(86-95)\end{array}$ & $\begin{array}{c}77 \\
(71-82)\end{array}$ & $\begin{array}{c}100 \\
(100-100)\end{array}$ & $\begin{array}{c}100 \\
(100-100)\end{array}$ & $0,14-1,0$ & $0,10-1,0$ \\
\hline
\end{tabular}

$Q_{1}$ : primer cuartil; $Q_{3}$ : tercer cuartil; IKG: índice kappa general; IKE: índice kappa de especies 
Cuadro 5. Resultados de los laboratorios intermedios en el programa de evaluación externa del desempeño

\begin{tabular}{cccccccccc}
\hline $\begin{array}{c}\text { Código del } \\
\text { laboratorio } \\
\text { intermedio }\end{array}$ & Resultado & Especie & Estadio & Recuento & IKG & IKE & \% VPPv & $\%$ VPPf & $\%$ VPIM \\
\cline { 2 - 5 }$n$ & 100,0 & 96,0 & 93,0 & 68,0 & 1,0 & 0,89 & 100 & 85,7 & 100,0 \\
31 & 100,0 & 96,0 & 86,0 & 66,0 & 1,0 & 0,89 & 100 & 85,7 & 100,0 \\
33 & 100,0 & 96,0 & 86,0 & 70,0 & 1,0 & 0,89 & 100 & 85,7 & 100,0 \\
Promedio $\left(I_{95 \%}\right)$ & & & $88(79-98)$ & $68,0(64-72)$ & & & & & \\
\hline
\end{tabular}

IKG: índice kappa general; IKE: índice kappa de especies; VP: valor predictivo; Pv: $P$. vivax; Pf: $P$. falciparum; IM: infección mixta $(P v+P f)$.

60

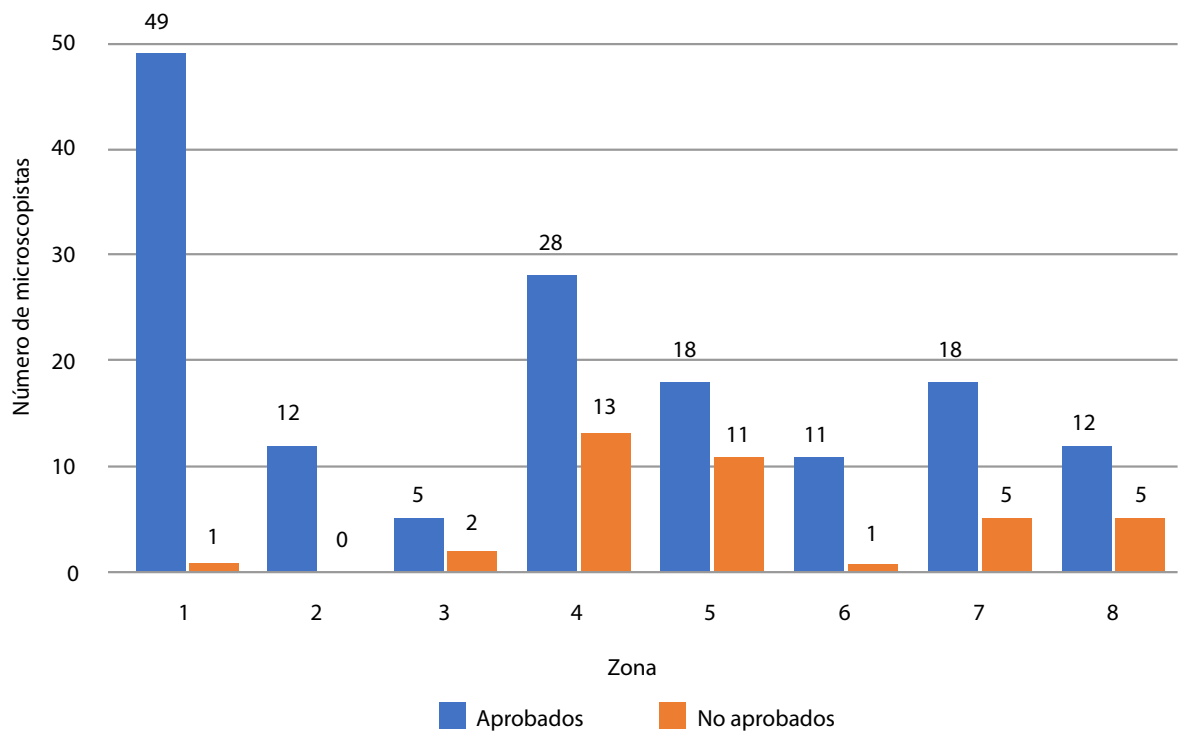

Figura 1. Microscopistas del nivel local aprobados y no aprobados en la evaluación de idoneidad por zona

para hacer el diagnóstico en los sitios de trabajo. Las actualizaciones del entrenamiento, por su parte, tienen como objetivo refrescar conocimientos y no dejar perder las habilidades adquiridas. Además, el programa de evaluación externa del desempeño es de gran importancia, ya que permite hacer seguimiento de la calidad del diagnóstico de los microscopistas en su sitio de trabajo mediante una evaluación anual entre laboratorios que permite garantizar la calidad de los resultados (6).

Los dos tipos de actividades cuentan con indicadores que son útiles para el análisis de la red de diagnóstico de malaria y para los análisis locales, nacionales e, incluso, regionales. La evaluación de los indicadores orienta la implementación de las medidas de mejoramiento continuo del personal responsable del diagnóstico.

Como lo indican los resultados de una revisión sistemática de estudios de evaluación externa de la calidad en los países de África subsahariana (17), dichas intervenciones deben incluirse en los planes operativos de acción institucionales. Las actividades de control de calidad tienen relevancia en la lucha contra la malaria, especialmente en los países de baja transmisión o que están en vías de eliminarla donde la observación de las muestras de pacientes positivos es poco frecuente y, por ello, se pierde la habilidad para identificar el parásito o la especie infecciosa (7), como sucedió, por ejemplo, 
en la evaluación del desempeño en un área no endémica de Etiopía en que se presentaron casos importados con diagnóstico incorrecto (18). Asimismo, es necesario llevar a cabo las actividades de control de calidad a los establecimientos de salud distantes, por ser estos los que brindan atención a la población más vulnerable (18).

Al comparar los resultados de la evaluación de la idoneidad en las diversas zonas del país, se observó que aquellas sin transmisión de malaria y que no reportan casos, o que lo hacen esporádicamente en el transcurso del año, tuvieron los mayores porcentajes de personas no aprobadas: zona 4 $(13 ; 38 \%)$, zona $5(11 ; 38 \%)$, zona $8(5 ; 29 \%)$ y zona $7(5 ; 22 \%)(19,20)$.

En cuanto a las medianas de la evaluación de la idoneidad de los microscopistas del nivel local (concordancia de resultado: 100,0 \% y concordancia de especies: 100,0 \%), se encontró que los valores fueron superiores a los de evaluaciones similares en algunos países de la región. Por ejemplo, en Perú, la mediana para la concordancia de resultados en microscopistas con experiencia fue de $70 \%$ y en las muestras positivas la más alta obtenida fue para $P$. vivax, con una mediana de $80 \%$; y en Colombia, el promedio en la concordancia de resultados fue de $96,9 \%$ y el de la concordancia de especies, de 88,7\% $(21,22)$.

Estas cifras se explicarían por los entrenamientos anuales que se organizan en Ecuador desde hace tres años para integrantes de la red de diagnóstico en zonas priorizadas por su perfil epidemiológico y que fueron liderados por el Instituto Nacional de Investigación en Salud Pública en coordinación con el Ministerio de Salud Pública y el apoyo de la OPS. La importancia del entrenamiento previo en el diagnóstico y el control de calidad guarda relación con los buenos resultados que se obtuvieron en las evaluaciones del diagnóstico hecho por los microscopistas, lo que coincide con los hallazgos de los estudios de Etiopía y Perú al comparar la calidad del diagnóstico de los microscopistas entrenados con los de aquellos que no lo estaban $(21,23)$; asimismo, la calidad del diagnóstico está relacionada con la experiencia de los microscopistas (21). Cabe resaltar que en el presente estudio la mayoría de los asistentes eran microscopistas con amplia experiencia en el diagnóstico de malaria.

Al analizar los resultados del número de microscopistas que aprobaron la concordancia de resultado (186) y la concordancia de especies (154) en la evaluación de la idoneidad, se observó una mayor dificultad para diferenciar la especie parasitaria infecciosa que para diferenciar entre muestras negativas y positivas, lo que concuerda con los resultados de otros estudios en estas mismas variables $(18,22)$.

En los componentes que tuvieron evaluación preliminar y posterior, es decir, el aspecto teórico, la concordancia de resultados y la concordancia de especies, se observó un cambio estadísticamente significativo entre las dos pruebas, excepto en la evaluación de resultados de las zonas 2, 3, 4, 6 y 7 y en la evaluación de especies en las zonas 3 y 7 , lo que significa que la mayoría de los participantes obtuvo buenos resultados en ambas pruebas.

Se encontró que las zonas del país más deficientes fueron la 4 y la 5, en las cuales se registró el mayor número de participantes que no aprobaron las evaluaciones de desempeño y con los índices kappa más bajos, lo cual exige priorizar estas zonas para implementar las medidas de intervención y seguimiento que fortalezcan el diagnóstico; de otra forma, se afectaría la 
selección del tratamiento adecuado y oportuno de los pacientes, así como la estrategia de eliminación de la malaria $(4,5,7)$.

Las concordancias de los resultados y de especies fueron los indicadores seleccionados para aprobar la evaluación de la idoneidad por considerarse variables determinantes para el diagnóstico. Las concordancias de estadio y de recuento tienen menos impacto en el resultado y se afinan con las actividades de control de calidad e intervenciones regulares (6).

En este orden de ideas, se destaca la importancia de la actualización del entrenamiento con evaluación de la idoneidad, la cual se hizo anualmente durante el periodo de estudio, lo que permitió demostrar que es posible obtener indicadores comparables con los de los estudios de la evaluación de la idoneidad en el Asia y el Pacífico (24).

El fortalecimiento de la capacidad del lector para diferenciar las láminas positivas con parasitemias bajas de las muestras negativas, además de la observación de las claves morfológicas y la idoneidad técnica de la elaboración y coloración ajustadas a los lineamientos internacionales $(6,25)$, son primordiales para evitar la subestimación de casos o la obtención de falsos positivos que conllevan el tratamiento excesivo de los pacientes, lo que puede conducir, en algunos casos, a agotar los antimaláricos en los establecimientos de salud y a aumentar la probabilidad de aparición de cepas resistentes a los medicamentos (24).

En la actualización de los entrenamientos es adecuado retomar las debilidades detectadas, como se hizo en el presente estudio en el que el principal error en la identificación de especies consistió en confundir $P$. falciparum con $P$. vivax cuando el primero presenta trofozoítos grandes.

Entre las actividades del país para garantizar la calidad del diagnóstico de malaria, se encuentran el entrenamiento, el programa de evaluación externa del desempeño, la evaluación indirecta del desempeño, la certificación de la idoneidad de los referentes por parte del nivel nacional y, en menor medida, las supervisiones (6).

Sin embargo, es necesario recordar que todas las actividades de calidad se sustentan en el control interno de calidad, el cual debe ser garantizado por cada laboratorio que ofrezca el servicio de diagnóstico parasitológico, lo que significa que deben contar con equipos que cumplan con las características técnicas y de mantenimiento, con reactivos para el diagnóstico microscópico, así como registros, procedimientos operativos estandarizados y suministro adecuado de consumibles (26-28). El control interno de calidad se destaca como parte integral del programa de control de calidad en los estudios que evalúan factores con impacto en la calidad del diagnóstico microscópico de malaria, en los que se han encontrado deficiencias importantes en la calidad y la preparación de reactivos, lineamientos, materiales e insumos y procedimientos básicos para la toma de muestras, su coloración y lectura, y en el reporte de resultados (23,29-31).

En un contexto más amplio, es necesario evaluar cuidadosamente el acceso al diagnóstico de malaria de las poblaciones en riesgo, pues se les debe garantizar el tratamiento antimalárico que elimine la condición de reservorio de los humanos en el ciclo de transmisión del parásito (5). En este contexto, las pruebas de diagnóstico rápido son complementarias del diagnóstico microscópico, siempre y cuando se realicen en el marco de un sistema que garantice su calidad $(7,17,32)$. 
Por otra parte, el desempeño alcanzado por los laboratorios intermedios fue satisfactorio en cuanto a las concordancias de resultado, especie y estadio; sin embargo, es necesario continuar organizando actividades para mejorar la concordancia de recuento en los laboratorios de referencia. En este sentido, los resultados del presente estudio contrastan con los reportados en otros en los que se hizo la evaluación externa del desempeño de los responsables del diagnóstico de los laboratorios locales sin entrenamiento o cuando este se ofrecía con poca frecuencia $(18,23)$.

Si bien en este estudio el número de laboratorios de nivel intermedio fue bajo, es importante enfatizar que, en la concordancia de resultado, los aciertos fueron del $100 \%$ y, en la concordancia de especies, del $96 \%$, superiores a los encontrados en evaluaciones de la región y de África occidental $(14,33)$. Esto podría explicarse por la amplia experiencia de los referentes y los entrenamientos programados para este nivel. Para mantener el nivel alcanzado, el laboratorio nacional debe seguir ofreciendo regularmente los entrenamientos y otras actividades dirigidas a los responsables del diagnóstico, así como brindar apoyo en las actividades organizadas por el nivel intermedio para la red primaria.

La deficiencia detectada en los referentes del nivel intermedio en cuanto al diagnóstico de la infección mixta como una infección por $P$. faciparum es frecuente, incluso en microscopistas referentes de otros países (14), pero debe corregirse haciendo una revisión más rigurosa que incluya un mayor número de campos microscópicos para, así, evidenciar la baja parasitemia de $P$. vivax, ya que, si se extrapola este error al diagnóstico de rutina, se estaría dando pie a posibles recaídas de los pacientes.

El indicador con mayor dificultad para los laboratorios intermedios fue la concordancia de recuento, tal vez debido a que la mayoría de las muestras positivas (10: $71 \%$ ) tuvieron parasitemias inferiores a 700 parásitos/ $\mu \mathrm{l}$, lo que refleja el grado de complejidad adecuado de las evaluaciones de idoneidad y desempeño cuando se trata de fortalecer el diagnóstico de bajas parasitemias (4).

En cuanto al programa de evaluación externa de desempeño, es necesario implementarlo en el nivel local. Sin embargo, su sostenibilidad representa un reto importante que requiere los recursos, la obtención de muestras suficientes, y el transporte seguro y oportuno, sobre todo en los centros de diagnóstico ubicados en lugares de difícil acceso, como se evidenció en el estudio sobre la implementación de la evaluación externa de la calidad nacional para los laboratorios médicos en Burkina Faso (33). El programa de evaluación externa del desempeño es una actividad de mucha relevancia para aquellos laboratorios que se encuentran en proceso de acreditación (33).

La obtención de los paneles de láminas es una labor difícil para los países con baja transmisión de malaria, como es el caso de Ecuador, por lo que el programa de evaluación externa del desempeño podría implementarse con microscopía virtual mediante el escaneo de muestras problemáticas, material que puede utilizarse para dar cobertura a todos los participantes de la red con acceso a un computador y a internet. Dicha metodología permite racionalizar la elaboración de lotes con un número adecuado de muestras y garantiza la homogeneidad de los paneles utilizados en la evaluación. Sin embargo, es necesario adquirir equipos y programas adecuados y estandarizar la metodología para que el laboratorio referente obtenga las imágenes escaneadas. Asimismo, es posible distribuir material escaneado como material de referencia para que los microscopistas tengan presente la morfología de las especies parasitarias $(34,35)$. 
El control de la calidad del diagnóstico debe ser un proceso sostenible y dinámico que garantice un diagnóstico confiable para el manejo adecuado de los pacientes con malaria según los lineamientos nacionales (36), y que permita el seguimiento de los casos, el apoyo en la vigilancia epidemiológica, y un diagnóstico oportuno y de calidad de los casos importados, incluso, en situaciones en las que no haya transmisión, como se demostró en la evaluación realizada en China durante la transición de un programa de control a uno de eliminación (37).

En cuanto a las limitaciones del estudio, puede mencionarse el hecho de que no se incluyeron todas las zonas de salud establecidas por el Ministerio de Salud Pública. Es el caso de la zona 9, una zona no receptiva y sin vulnerabilidad donde se había realizado la misma actividad ocho meses antes. Tampoco se evaluaron la elaboración y la coloración de las muestras por limitaciones de tiempo y recursos.

En conclusión, este es el primer estudio nacional en que se analizan los resultados de dos actividades de control de la calidad del diagnóstico microscópico de malaria en dos niveles de la red de laboratorios. Se demostró que los indicadores de idoneidad de la red de diagnóstico primaria y los de desempeño de los laboratorios intermedios, alcanzaron altos estándares de calidad, lo que responde al programa de entrenamiento implementado en el país.

\section{Agradecimientos}

Los autores expresan su agradecimiento a las comunidades que viven en las zonas de transmisión endémica de malaria, a los niveles central, zonal y local del Ministerio de Salud Pública y a la OPS.

\section{Referencias}

1. World Health Organization. Eliminating malaria. Geneva: World Health Organization; 2016. Fecha de consulta: 18 de abril de 2018. Disponible en: https://www.who.int/malaria/ publications/atoz/eliminating-malaria/en/

2. Ministerio de Salud Pública de Ecuador. Reformar el reglamento interno para la administración y control de activos fijos del Ministerio de Salud Pública. Fecha de consulta: 20 de abril de 2018. Disponible en: https://aplicaciones.msp.gob.ec/salud/archivosdigitales/ documentosDirecciones/dnn/archivos/ac 00005108201402 set.pdf

3. Presidencia de la República de Ecuador. Decreto Ejecutivo No. 1290/2012 de 30 de agosto. Fecha de consulta: 20 de abril de 2018. Disponible en: http://www.controlsanitario.gob.ec/wpcontent/uploads/downloads/2014/04/DECRETO-EJECUTIVO-DE-CREACION-DEL-ARCSA.pdf

4. Tangpukdee N, Duangdee C, Wilairatana P, Krudsood S. Malaria diagnosis: A brief review. Korean J Parasitol. 2009;47:93-102. https://doi.org/10.3347/kjp.2009.47.2.93

5. World Health Organization. Guidelines for the treatment of malaria. Geneva: World Health Organization; 2015. p. 317. Fecha de consulta: 23 de febrero de 2018. Disponible en: http:// www.who.int/malaria/publications/atoz/9789241549127/en/_

6. World Health Organization. Malaria microscopy quality assurance manual. Version 2. Geneva: World Health Organization; 2016. p. 121.

7. World Health Organization. A framework for malaria elimination. Geneva: World Health Organization; 2017. p. 100. Fecha de consulta: 23 de febrero de 2018. Disponible en: http:// www.who.int/malaria/publications/atoz/9789241511988/en/

8. Poostchi M, Silamut K, Maude RJ, Jaeger S, Thoma G. Image analysis and machine learning for detecting malaria. Transl Res. 2018;194:36-55. https://doi.org/10.1016/j.trsl.2017.12.004

9. World Health Organization. Universal access to malaria diagnostic testing: An operational manual. Geneva: WHO; 2011. p. 160. Fecha de consulta: 5 de febrero de 2018. Disponible en: http://www.who.int/malaria/publications/atoz/9789241502092/en/ 
10. World Health Organization. World malaria report, 2017. Geneva: World Health Organization; 2017. p. 196. Fecha de consulta: 5 de febrero de 2018. Disponible en: http://www.who.int/ malaria/publications/world-malaria-report-2017/en/

11. Moakofhi K, Edwards JK, Motlaleng M, Namboze J, Butt W, Obopile M, et al. Advances in malaria elimination in Botswana: A dramatic shift to parasitological diagnosis, 2008-2014. Public Health Action. 2018;8:S34-8. https://doi.org/10.5588/pha.17.0017

12. World Health Organization. Policy brief on malaria diagnostics in low-transmission settings. Fecha de consulta: 25 de febrero de 2018. Disponible en: http://www.who.int/malaria/ publications/atoz/policy-brief-diagnosis-low-transmission-settings/en

13. Foundation for Innovative New Diagnostics. Malaria rapid diagnostic tests. An implementation guide. Geneva: Foundation for Innovative New Diagnostics; 2013. p. 88. Fecha de consulta: 15 de febrero de 2018. Disponible en: https://www.gov.uk/government/uploads/system/ uploads/attachment_data/file/243817/malaria_rdt_implementation_guide2013.pdf

14. Organización Panamericana de la Salud. Informe técnico, cuarto panel, 2014-2015. Programa de evaluación externa del desempeño para el diagnóstico microscópico de la malaria. Fecha de consulta: 27 de abril de 2018. Disponible en: http://www.paho.org/hq/ index.php?option=com_docman\&task $=d o c \_d o w n l o a d \& l t e m i d=\& g i d=32909 \&$ lang $=e s$

15. Gutiérrez S, Arróspide N. Manual de procedimientos de laboratorio para el diagnóstico de malaria. Lima: Ministerio de Salud, Instituto Nacional de Salud; 2012. p. 46. Fecha de consulta: 15 de abril de 2018. Disponible en: http://www.ins.gob.pe/repositorioaps/0/4/jer/1/ Antimalaricos/manualMALARIA.pdf

16. Organización Panamericana de la Salud. Programa de evaluación externa del desempeño para el diagnóstico microscópico de malaria. Fecha de consulta: 10 de febrero de 2018. Disponible en: http://linksglobal.org/AMI/extras/PEED_Malaria_OPS_Esp_2011.pdf

17. Carter JY. Special issue: External quality assessment in laboratory medicine. External quality assessment in resource-limited countries. Biochem Med. 2017;27:97-109. https://doi.org/10.11613/BM.2017.013

18. Yitbarek T, Nega D, Tasew G, Taye B, Desta K. Performance evaluation of malaria microscopists at defense health facilities in Addis Ababa and its surrounding areas, Ethiopia. PLoS One. 2016;11:e0166170. https://doi.org/10.1371/journal.pone.0166170

19. Subsecretaría Nacional de Vigilancia de la Salud Pública. Enfermedades transmitidas por vectores. Informe malaria. Semana epidemiológica (SE) 01/2/2017. Ecuador. Fecha de consulta: 10 de noviembre de 2018. Disponible en: https://www.salud.gob.ec/wpcontent/ uploads/2017/07/Gaceta-Vectorial-SE52.pdf

20. 20. Dirección Nacional de Vigilancia Epidemiológica. Anuario de vigilancia epidemiológica 2017. Enfermedades transmitidas por vectores. Fecha de consulta: 10 de noviembre de 2018. Disponible en: https://public.tableau.com/profile/vvicentee80\#!/vizhome/ EnfermeddaesTropicales vectoriales-2014/ANUARIO

21. Rosas-Aguirre A, Gamboa D, Rodríguez H, Llanos-Zavalaga F, Aguirre K, Llanos-Cuentas A. Uso de paneles de láminas estandarizadas para la evaluación de competencias en el diagnóstico microscópico de malaria en la amazonía peruana. Rev Peru Med Exp Salud Pública. 2010;27:540-7.

22. Mendoza NM, González NE. Evaluación del desempeño mediante paneles de láminas: una herramienta para la clasificación de los microscopistas senior del Programa de Control de la Malaria en Colombia. Biomédica. 2015;35:582-9. https://doi.org/10.7705/biomedica.v35i4.2694

23. Sori G, Zewdie O, Tadele G, Samuel A. External quality assessment of malaria microscopy diagnosis in selected health facilities in Western Oromia, Ethiopia. Malar J. 2018;17:233. https://doi.org/10.1186/s12936-018-2386-2

24. Ashraf S, Kao A, Hugo C, Christophel EM, Fatunmbi B, Luchavez J, et al. Developing standards for malaria microscopy: External competency assessment for malaria microscopists in the Asia-Pacific. Malar J. 2012;11:352. https://doi.org/10.1186/1475-2875-11-352

25. World Health Organization. Basic malaria microscopy. Part I. Learner's guide. Geneva: World Health Organization; 2010. p. 83. Fecha de consulta: 10 de abril de 2018. Disponible en: http://www.who.int/malaria/publications/atoz/9241547820/en/

26. World Health Organization. Preparation of water buffered to $\mathrm{pH}$ 7.2. Malaria microcopy standard operating procedure-MM-SOP-03A. 2016. Fecha de consulta: 17 de enero de 2018. Disponible en: http://www.wpro.who.int/mvp/lab_quality/2096_oms_gmp_sop_03a_rev.pdf 
27. World Health Organization. Preparation of Giemsa working solution. Malaria microscopy standard operating procedure- MM--SOP-04. 2016. Fecha de consulta: 17 de enero de 2018. Disponible en: http://www.wpro.who.int/mvp/lab_quality/2096_oms_gmp_sop_04_rev.pdf

28. World Health Organization. Cleaning and storing microscope slide. Malaria microscopy standard operating procedure- MM-SOP-01. 2016. Fecha de consulta: 17 de enero de 2018. Disponible en: http://www.wpro.who.int/mvp/lab quality/2096 oms gmp sop 01 rev.pdf

29. Wanja E, Achilla R, Obare P, Adeny R, Moseti C, Otieno V, et al. Evaluation of a laboratory quality assurance pilot programme for malaria diagnostics in low transmission areas of Kenya, 2013. Malar J. 2017;16:221. https://doi.org/10.1186/s12936-017-1856-2

30. Biadglegne F, Belyhun Y, Ali J, Walle F, Gudeta N, Kassu A, et al. Does the practice of blood film microscopy for detection and quantification of malaria parasites in northwest Ethiopia fit the standard? BMC Health Serv Res. 2014;14:529. https://doi.org/10.1186/s12913-014-0529-x

31. Abreha T, Alemayehu B, Tadesse Y, Gebresillassie S, Tadesse A, Demeke L, et al. Malaria diagnostic capacity in health facilities in Ethiopia. Malar J. 2014;13:292. https://doi.org/10.1186/1475-2875-13-292

32. World Health Organization. Good practices for selecting and procuring rapid diagnostic tests for malaria. Geneva: World Health Organization; 2011. p. 108. Fecha de consulta: 25 de febrero de 2018. Disponible en: http://www.who.int/malaria/publications/atoz/9789241501125/en

33. Sakandé J, Nikièma A, Kabré E, Sawadogo $\mathrm{CH}$, Nacoulma EW, Sanou M, et al. Implementation of a national external quality assessment program for medical laboratories in Burkina Faso. Am J Clin Pathol. 2014;141:181-7. https://doi.org/10.1309/AJCPXC83HIBBSRDT

34. Ahmed L, Seal LH, Ainley C, De la Salle B, Brereton M, Hyde K, et al. Web-based virtual microscopy of digitized blood slides for malaria diagnosis: An effective tool for skills assessment in different countries and environments. J Med Internet Res. 2016;18:e213. https://doi.org/10.2196/jmir.6027

35. Abdul-Ghani R. Towards e-parasitology: Making use of virtual microscopy. Trop Med Int Health. 2015;20:227-9. https://doi.org/10.1111/tmi.12426

36. Ministerio de Salud Pública. Manual de procedimientos del Subsistema de Vigilancia Epidemiológica Alerta Acción SIVE-ALERTA. Quito: Ministerio de Salud; 2013. Fecha de consulta: 2 de mayo de 2018. Disponible en: https://aplicaciones.msp.gob.ec/salud/archivosdigitales/ documentosDirecciones/dnn/archivos/manual de procedimientos sive-alerta.pdf

37. Sun JL, Zhou S, Geng QB, Zhang Q, Zhang ZK, Zheng CJ, et al. Comparative evaluation of the diagnosis, reporting and investigation of malaria cases in China, 2005-2014: Transition from control to elimination for the national malaria programme. Infect Dis Poverty. 2016;5:65. https://doi.org/10.1186/s40249-016-0163-4 